\title{
Considerations for the Use of Medical Cannabis: An Overview of Benefits and Harms
}

\author{
Dhana Ratna Shakya*1, Sandesh Raj Upadhaya ${ }^{1}$, Hari Neupane ${ }^{2}$ and Roshan Subedi ${ }^{3}$ \\ ${ }^{1}$ Department of Psychiatry, BP Koirala Institute of Health Sciences, Dharan, Nepal \\ ${ }^{2}$ Health Foundation Nepal, Jandragaun, Ghorahi, Nepal \\ ${ }^{3}$ Nepal Health Research Council, Kathmandu, Nepal \\ *Corresponding author: Dhana Ratna Shakya, Department of Psychiatry, BP Koirala Institute of Health Sciences, Dharan, Nepal
}

\section{ARTICLE INFO}

Received: 蔧 June 10, 2021

Published: 业 June 21, 2021

Citation: Dhana Ratna Shakya, Sandesh Raj Upadhaya, Hari Neupane, Roshan Subedi. Considerations for the Use of Medical Cannabis: An Overview of Benefits and Harms. Biomed J Sci \& Tech Res 36(4)2021. BJSTR. MS.ID.005886.

Keywords: Cannabis; Cannabis use; Benefit; Harm; Adverse effect

\section{STRUCTURED ABSTRACT}

Introduction: Cannabis use to treat various diseases or symptoms is not clearly defined. The purpose of this review is to explore the existing evidence regarding the harms and benefits of cannabis use across multiple illnesses.

Material and Methods: Search for articles for this review was performed in PubMed, Google Scholar and PsycINFO. We summarize and discuss recent evidence regarding cannabis use in multiple neurological (including seizures, neurodegenerative disorders, multiple sclerosis), psychiatric (including depression, bipolar disorder, anxiety and psychotic illness) and other diseases (including chronic pain, cancer, vomiting, cardiovascular diseases, COVID-19).

Results: The collated body of evidence shows that the benefit of its use (medicinal or recreational) mostly lacks adequate evidence warranting for further study. In pretext of already available better options (with better efficacy and safety), the consideration of this agent is seriously debatable.

Conclusion: Harms or adverse effects of cannabis use clearly are evidence based and are at times serious (e.g. psychosis association), mainly from mental health perspective. Hence, its use should not be suggested and needs serious consideration before using it.

\section{Introduction}

Cannabis are products of the plants Cannabis Sativa and Cannabis Indica [1]. Cannabis is the most abused illicit drug and is cultivated in 151 nations [2]. Cannabinoids are major pharmacologically active compounds in cannabis which exist mainly as: endocannabinoids, phyto-cannabinoids and synthetic cannabinoids [3]. Endocannabinoids (like anandamide), synthesized by human body, act on cannabinoid receptors (CB): CB1 (brain) and CB2 (immune system) of endocannabinoid system which plays a key role in maintaining body homeostasis. Phyto-cannabinoids are exogenous plant-derived cannabinoids concentrated in oil resin of the buds and the leaves of cannabis plants with affinity for mammalian cannabinoid receptors. Of more than 113 cannabinoids known, tetrahydrocannabinol (THC, a major psychoactive compound) and cannabidiol (CBD, showing therapeutic potential) are major Phyto cannabinoids [4-6]. Synthetic Cannabinoids (like nabilone) are labmade molecules designed to mimic Phyto-cannabinoids action. To date, Food and Drug Administration (FDA) has approved one cannabis-derived drug product (direct plant extract): Epidiolex (cannabidiol) and three synthetic cannabis-related products (synthetic isolates): Marinol (dronabinol), Syndros (dronabinol) and Cesamet (nabilone) [7].

Cannabis use is either 'medical' (treating symptoms/ conditions) or 'recreational' (getting high) [8]. Cannabis is kept under schedule-I (research and medical) drug and is prohibited 
for growing, buying and selling for recreational use at the federal level in the United States (US). However, its 36 states, four territories and the District of Columbia have legalized its medical use. Worldwide, more than three dozen nations have legalized medical cannabis use as of 2020. The United Nations' Single Convention on Narcotic Drugs, 1961 included cannabis under schedule-IV (under the strictest control schedule) and discouraged its medical use. Based on the World Health Organization (WHO) recommendation to remove cannabis from schedule-IV of the 1961 convention, the Commission on Narcotics drugs decided to follow this recommendation in December 2020, paving the way for its therapeutic use. Recreational and other uses are still illegal [9]. Changing dynamics in research at times demonstrate its usefulness for medical purpose due to anxiolytic, neuroprotective, antioxidant, anti-inflammatory, antidepressant, antipsychotic and hypnotic actions [10].

Cannabis is consumed mainly as: smoking cigarettes (joints) or inhaling from pipes, vaporizer, or resin burnt in a sawn-off plastic bottle. It can also be eaten by baking into cookies or cakes; edible form as bhang in Indian subcontinent and occasionally drunk as an extract [11]. The Global burden of disease estimates- 2019 suggests cannabis use disorders resulting in 690,000 global years lost due to disability, $0.08 \%$ (0.05-0.1\%) of total global YLDs [12]. Opponents of legalization view cannabis as a harmful substance due to its abuse potential, risk of aggravation of psychosis, road traffic accidents, low birth weight and respiratory diseases. Many studies are mostly inconclusive regarding cannabis use, with only a few studies showing some benefits in certain conditions (pain, nausea, vomiting, spasticity). These conclusions are also not properly interpreted, communicated and reported to policymakers, care providers and stakeholders, which has a huge impact on public health $[8,13]$. This narrative review intends to explore the existing evidence regarding harms and benefits of cannabis across multiple physical and mental problems.

\section{Method}

This is a narrative review evaluating literature obtained using electronic search in databases of PubMed, Google Scholar and PsycINFO, published till May 2021. The following keywords were used in different combinations for the search: 'cannabis', 'medical cannabis', 'cannabidiol', 'benefits', 'use', 'indication', 'harm', 'side effects', 'adverse effects', 'physical health' and 'mental health'. The search strategy used in PubMed was: (Medical cannabis [Title/ Abstract]) AND (Benefit [Title/Abstract] OR harm [Title/Abstract] OR use [Title/Abstract] OR indication [Title/Abstract] OR adverse effect [Title/Abstract] OR side effect [Title/Abstract] OR physical health [Title/Abstract] OR mental health [Title/Abstract]). Studies (cross-sectional, cohort, case-control, clinical trial, and reviews) relating to the benefits and harms of medical cannabis were included. The included articles were evaluated by the reviewers to extract relevant information regarding its benefits and harms.

\section{Neurological Disorders}

Cannabis is being explored for treatment of several neurological disorders [multiple sclerosis (MS), Parkinson's disease (PD), Alzheimer's disease, Huntington's disease, neuropathic pain, epilepsy] [14].

\section{Epilepsy and Refractory Seizures}

CBD has been reported with anti-seizure properties, which are not direct action on cannabinoid receptors but are mediated through several other mechanisms, like agonist or antagonist effects on ionic channels, neurotransmitter transporters and multiple 7-transmembrane receptors [15]. A systematic review [four randomized trials involving 550 patients with Lennox-Gastaut syndrome (LGS) and Dravet syndrome (DS)] showed greater reduction in seizure frequency with oral CBD [15]. The pooled average difference in change in seizure frequency during treatment period was 19.5\% [95\% confidence interval (CI) 8.1-31.0] with CBD $10 \mathrm{mg}$ and $19.9 \%$ (95\% CI: 11.8-28.1) with CBD 20mg, compared to placebo-group. Reduction in all types of seizure frequency by at least $50 \%$ occurred in $37.5 \%$ of participants using $20 \mathrm{mg}$ Cannabinoid in comparison to $21.2 \%$ in placebo-group [Risk ratio (RR): 1.76 ; $95 \%$ CI 1.07-2.88]. In June 2018, FDA approved Epidiolex, a purified form of CBD, for the treatment of seizures of Lennox-Gastaut syndrome, Dravet syndrome and tuberous sclerosis in patients one year of age and older [16]. A study from Nepal reports $4 \%$ of seizure psychiatry out-patients to abuse cannabis [17].

\section{Neurodegenerative Disorders}

CBD displays neuroprotective role via several mechanisms, i.e., through different signal transduction pathways mediated indirectly by cannabinoid receptors. It prevents glycogen synthase kinase $3 \beta$ (GSK-3 $\beta$ ) hyperphosphorylation caused by $A \beta$, making it a new therapeutic candidate for Alzheimer's disease [18]. A systematic review (twelve primary studies and one uncontrolled before-andafter prospective pilot study) suggests cannabis as effective for treating neuropsychiatric symptoms associated with dementia, e.g., agitation, disinhibition, irritability, nocturnal behavior disorders, aberrant motor behavior and aberrant vocalization [19]. Dementia is a qualifying condition for medical cannabis use in ten $(41.7 \%)$ of the 24 states and localities in the US where medical marijuana is legal, primarily for agitation of Alzheimer's disease [20].

A small study among 21 PD patients without dementia or comorbid psychiatric conditions indicates possible effect of CBD in improving quality of life measures. The groups treated with placebo and CBD 300mg/day had significantly different mean total scores in the Parkinson's Disease Questionnaire-39 (p-value= 0.05) [21]. 
It warranted additional studies with larger samples and specific objectives to establish its benefit.

A double-blind, randomized, placebo-controlled, crossover pilot clinical trial with botanical extract of cannabis for the treatment of Huntington's disease, shows no differences on motor ( $p$-value $=0.286)$, cognitive $(p$-value $=0.824)$, behavioral $(p$-value $=$ 1.0 ) and functional ( $p$-value $=0.581$ ) scores as compared to placebo [22].

\section{Multiple Sclerosis (MS) and spasticity}

MS is characterized by auto-immune-mediated CNS myelin sheath destruction resulting in upper motor neuron lesion with features of muscle spasticity. Evidence supports baclofen, tizanidine, and gabapentin as first-line management of its spasticity [23]. A double-blind, placebo-controlled, phase-III study among 274 MS patients showed significant effects of cannabis extract in body pain, spasms and sleep quality [assessed with Category Rating Scale (CRS)] [24]. The rate of relief from muscle stiffness after 12 weeks (2-week dose-titration phase from $5 \mathrm{mg}$ to a maximum of $25 \mathrm{mg}$ of THC daily and a 10-week maintenance phase) was almost twice with cannabis extracts as with placebo [29.4\% vs $15.7 \%$; odds ratio (OR): 2.26; 95\% CI: 1.24-4.13]. Other randomized double-blinded study among patients with definite MS and spasticity demonstrated whole-plant cannabis-based medicine containing $\Delta-9$ THC and CBD active preparation as significantly superior to placebo ( $\mathrm{p}$-value= 0.048) [25]. A meta-analysis of five trials found cannabinoids with a greater average improvement on the Ashworth Scale for spasticity compared with placebo in patients with spasticity due to MS or paraplegia, although the difference was statistically not significant $(-0.12$; 95\% CI: -0.24 to 0.01$)$ [26].

\section{Psychiatric Disorders}

\section{Psychotic disorder and Schizophrenia}

Cannabis use has been shown to alter the age of onset, course and presentation of psychotic disorder in vulnerable individuals [27]. A systematic review of 35 longitudinal population-based studies shows any cannabis use to associate with an increased risk of psychosis: adjusted odds ratio (AOR) of 1.41. There was significant dose-response relationship with twice the risk (RR: 2.09) for using cannabis more often [28]. Different factors increase the psychosis risk: type of cannabis (with high THC/CBD ratio), vulnerable subpopulation (adolescents), and familial and genetic factors (including the AKT1 rs2494732 polymorphism) [29]. After the onset of psychosis too, continued cannabis use is associated with more adverse outcomes, i.e., increased relapserate, prolonged hospital-stay, severe positive symptoms [30]. Several studies show increased risk of schizophrenia with its use and cannabis-induced psychosis. In the population-based study from Danish Civil Registration System and Psychiatric Central
Research Register, $41.2 \%$ of 1,492 cannabis-induced psychosis patients were ultimately diagnosed with schizophrenia. Half of the male patients with a cannabis-induced psychosis converted within 2.0 years and rest half of the female patients converted within 4.4 years [31]. Results are equivocal with CBD (1000mg) in one clinical trial showing efficacy with a reduction in positive symptoms and improving functional outcome in schizophrenia and at next dose of $600 \mathrm{mg}$ did not show being better than placebo in improving symptoms and cognition in stable treated schizophrenia patients. Current data are nascent regarding any benefit of CBD in psychosis [32].

\section{Mood Disorders}

Depression: Meta-analysis (with total size of 176,976) of nationally representative samples showed a three-fold increased risk of major depression in individuals with cannabis use disorder, particularly higher risk with cannabis dependence (pooled OR 4.83) [33]. A 2018 meta-analysis of 36 studies consisting of 19 crosssectional and 17 longitudinal studies showed a positive association between depression and cannabis use with an (OR: 1.29; 95\% CI: 1.10-1.51) among adolescents and young adults. There was also unidirectional relationship of cannabis use with depression (OR:1.33; 95\% CI: 1.19-1.49) [34]. There are no RCTs using CBD for treatment of depression [32]. Caution is required even while using Epidiolex (FDA-approved CBD), highlighting possible adverse reactions as depression and suicidal ideation [35].

Bipolar Disorder: A systematic review showed a positive relationship between cannabis use and aggravation of mania in diagnosed bipolar disorder (BPD). A meta-analysis of two studies showed approximately 3-fold (OR: 2.97; 95\% CI: 1.804.90) increased chance for new manic symptoms in people using cannabis [36]. A three-year prospective study found conflicting results, where weekly to almost daily use resulted in increased chance of BPD (AOR: 2.47; 95\% CI: 1.03-5.92), but not daily use (AOR: 0.52; 95\% CI: 0.17-1.55) [37]. Cannabis use is also related to poor prognosis with higher chance of rapid cycling and mixed episodes in bipolar patients [38]. There are no studies regarding CBD use for BPD treatment.

\section{Anxiety Disorder}

A cohort study on 112,000 non-institutionalized general population of 10 countries showed a positive association between anxiety and cannabis use (OR=1.24; 95\% CI: 1.06-1.45; 15 studies). The association with cannabis use disorder $(\mathrm{OR}=1.68$; $95 \% \mathrm{CI}$ : 1.23-2.31; 13 studies) was even higher [39]. A systematic review and meta-analysis of 24 longitudinal studies on baseline cannabis use and anxiety outcomes found that cannabis use may increase the odds of developing any anxiety condition (OR: 1.25; 95\% CI: 1.01-1.54). However, generalized anxiety disorder, panic disorder, or social anxiety disorder are not significantly associated with 
cannabis use [40]. A Nepali study reports panic disorder as the most common presentation among cannabis users [41] and other one reports $4 \%$ of OCD patients visiting a psychiatry clinic to abuse cannabis [42]. There is a low quality of evidence suggesting a single dose CBD 600mg given to individuals with social anxiety disorder showing improved scores on visual analog scale in simulated public speaking test in comparison to placebo [26]. More robust clinical trials are needed to validate its anti-anxiety effect.

\section{Neuropsychological Effects}

Harmful effect of cannabis on neurocognition is related to the preparation of cannabis with a higher THC:CBD ratio [43]. A systematic review and meta-analysis involving 69 studies found statistically significant negative effects in cognitive functioning with frequent or heavy cannabis use by adolescents and young adults, albeit the association was small. The abstinence for more than 72 hours decreased these cognitive deficits [44]. Other cohort study involving continued daily heavy cannabis use among adolescents to adults also showed a decline in executive functioning, learning, memory and verbal IQ at 38 years of age even after adjusting for multiple related covariates, and the neurocognitive functioning not improving in adolescent-onset cannabis use even after stopping cannabis [45].

\section{Psychosocial Consequences}

Adolescent cannabis use is associated with poorer psychosocial functioning resulting in lower educational attainment, risk of other drug use and higher levels of antisocial behavior during adulthood. In adults using cannabis frequently and for a prolonged time; poorer psychosocial functioning was reported in many domains (academic performance, educational attainment, unemployment, occupational prestige/income, financial strain, alcohol and other drug use, antisocial behavior and interpersonal relationships likefamily and social relationship [46]) regardless of whether it was adolescent-onset or adult-onset. Association is not clear and causal mechanism is more likely to be social milieu factors [47].

\section{Others}

\section{Chronic Pain}

Cannabis-based medicines (CBMs) are found to reduce chronic pain resulting from multiple causes. A systematic review and meta-analysis of 24 randomized controlled trials (a total of 1,334 patients) showed reduction in chronic pain $(-0.61 ; 95 \% \mathrm{CI}:-0.78$ to -0.43$)$, especially by inhalation $(-0.93$; $95 \% \mathrm{CI}$ : -1.51 to -0.35$)$ compared to placebo [48]. In other meta-analysis, cannabinoids had a greater average number of patients with a reduction in pain (37\% vs 31\%; OR: 1.41; 95\% CI: 0.99-2.00; 8 trials), and a greater average reduction in numerical rating scale pain assessment (on a 0-10-point scale; weighted mean difference: -0.46 ; $95 \%$ CI: -0.80 to $-0.11 ; 6$ trials) compared to placebo [26]. This analgesic property has made them an option for control of cancer pain.

\section{Cancer}

Cannabinoids have some antineoplastic potential. A systematic review of experimental studies performed on animal colorectal cancer models has shown a reduction in the formation of preneoplastic lesions (aberrant crypt foci) and volume of neoplastic lesions [49]. CBD botanical substances were reported to achieve high aberrant crypt foci (ACF) reduction (86\%) and tumor volume reduction via induction of apoptosis, interaction with cell survival, growth pathways and angiogenesis inhibition. Cannabis is also found to down-regulate immunologically generated free radical production by promoting a Th2 immune cytokine profile and hence, minimizes some carcinogenic pathways. THC inhibits the enzyme necessary to activate some of the carcinogens of smoke. Due to lack of cannabinoids receptors in respiratory epithelial cells of lungs, DNA damage checkpoint mechanism is reported to remain intact even after prolonged cannabis smoking [50]. A pooled analysis of six case-control studies with a total of 2,159 cases and 2,985 controls failed to prove cannabis smoking as a risk factor for lung cancer and reported the overall pooled OR for habitual versus non-habitual cannabis users or never-users 0.96 (95\% CI: 0.661.38) [51].

Several other studies have proven its role in carcinogenesis. A systematic review and meta-analysis of three case-control studies have found the strongest association between cannabis smoking and testicular germ cell tumor (TGCT). The TGCT risk is strongly associated with current cannabis use or using cannabis at least a week or long duration ( $>10$ years) with the pooled summary OR 1.62 (95 \% CI: 1.13-2.31), 1.92 (95 \% CI: 1.35-2.72), and 1.50 (95\% CI: 1.08-2.09) respectively [52].

\section{Cardiovascular System}

Cannabis smoking can result in cardiovascular adverse effects via sympathetic nervous system stimulation, parasympathetic nervous system inhibition, inflammation of blood vessels, platelet activation and carboxyhemoglobin generation [53]. Although rare, it is associated with an increased risk of life-threatening cardiac dysrhythmia [54]. A systematic review of published cases of individuals with acute myocardial infarction following marijuana use has reported episodic marijuana use as a significant risk factor for acute myocardial infarction, particularly in individuals with no cardiac risk factors [55].

\section{Reproductive System}

The active components of cannabis activate the cannabinoid receptors of internal reproductive organs in both sexes, a part of endogenous endocannabinoid system [56]. A study among 1,215 Danish men aged 18-28 years showed regular marijuana smoking >once/week to associate with 29\% (95\% CI: -46 to -1) lower sperm count after adjusting for confounders. If the same frequency of cannabis smoking were combined with the use of other recreational 
drugs, sperm concentration would be reduced by 52\% (95\% CI: -68 to -27 ) and sperm count by $55 \%$ (95\% CI: -71 to -31 ) [57]. This finding contradicted with other longitudinal study among 662 sub-fertile men at the Massachusetts General Hospital Fertility Center. In the latter study, current or past marijuana smokers had significantly higher sperm concentration (62.7; 95\% CI: 56.0 - 70.3 million $/ \mathrm{mL}$ ) than those who never smoked marijuana $(45.4 ; 95 \%$ CI: 38.6- 53.3 million/mL) [58].

Cannabis is found to affect the fertility of women via either ovulatory delay or inhibition. A study done in 201 participants showed that women with occasional marijuana users (up to three times in the last 3 months) and infrequent users (more than three times in the last 3 months) had a longer follicular phase than nonusers (3.5 days and 2 days longer respectively) [59]. Cannabis also affects the pregnancy, its outcome, and physical plus neuropsychiatric development of newborn. A systematic review and meta-analysis of 24 studies have demonstrated that cannabis use during pregnancy is associated with decreased birth weight of newborns when compared with infants of non-smokers [low birth weight pooled OR: 1.77; 95\% CI: 1.04 - 3.01] [60]. Cannabis use during pregnancy has been linked to a variety of neurobehavioral and cognitive effects in child; like inattention, impulsivity, learning and memory deficits and decline in executive functions [61].

\section{Ophthalmological}

Animal studies have shown the involvement of cannabinoids in retinal neurotransmission, neuroplasticity and neuroprotection. Cannabinoids can alter retinal signal and retinal synaptic plasticity, particularly during postnatal development. Cannabinoids are found to play a neuroprotective function by preventing retinal cell death; providing the support for development of synthetic cannabinoids as new therapeutic agents to prevent and treat retinal diseases $[62,63]$. The endocannabinoid system (ECS) has also been found to play a role in lowering intraocular pressure, making cannabis a potential therapeutic agent in managing glaucoma. However, its side effects, i.e., tolerance and accompanying psycho-active influence have largely limited this use $[64,65]$. A mice study has shown topical application of cannabis extract to reduce corneal hyperalgesia and neutrophil infiltration resulting from superficial chemical injury of corneal epithelium [66]. However, long-term cannabis use is associated with significant decrease in corneal cellular density. A study among 28 patients with cannabis use disorder and 32 age-and sex-matched healthy individuals, has shown a significant decrease $(\mathrm{p}<0.01)$ in corneal cell density (CD) in the cannabinoid group than control (mean CD was $2900 \pm 211$ cells $/ \mathrm{mm}^{2}$ vs. $3097 \pm 214$ cells $/ \mathrm{mm}^{2}$ ) [67].

\section{Hyperemesis Syndrome}

Cannabis acts both as pro-emetic and antiemetic agents. It exerts anti-emetic action at low doses and acts as pro-emetic at higher doses [15]. People with chronic cannabis use sometimes present with cyclic episodes of nausea and vomiting and get relief by frequent hot bathing. This syndrome is known as cannabis hyperemesis syndrome [68].

\section{Road Traffic Accidents}

Deterioration of specific cognitive functions and psychomotor abilities after cannabis use is responsible for impaired driving performance. The degree of impairment in performance observed in experimental studies with up to $300 \mathrm{mcg} / \mathrm{kg}$ THC was equivalent to the impairing effect of an alcohol dose producing a blood alcohol concentration of more than or equal to $0.05 \mathrm{~g} / \mathrm{dl}$, the legal limit for driving in most European countries. Blood THC levels do not correlate well with the level of cognitive impairment as it is quickly cleared from plasma and is widely distributed to tissues $[69,70]$. Use of both alcohol and cannabis has been shown to lead to greater odds of making an error than solo use of these substances [71].

\section{Sleep Disorder}

Although short-term THC use may show beneficial effects on reducing sleep latency, long-term use causes impaired sleep quality [72]. Sleep disruption and insomnia are associated with cannabis withdrawal, particularly in heavy cannabis use. In a study involving 30,000 teenagers in Fairfax County, Virginia with each hour of sleep lost, the chance of using marijuana increased by $23 \%$ suggesting the increasing use of marijuana after sleep deprivation $[1,73]$. Synthetic isolates like nabilone and dronabinol have shown some promise in obstructive sleep apnea (OSA) due to their modulatory effects on serotonin-mediated apneas in novel studies. For REMrelated sleep behavior disorder and excessive daytime sleepiness, CBD may be beneficial. Nabilone is useful in treating secondary insomnia conditions, e.g., PTSD and chronic pain. However, more robust evidence is needed to consolidate the findings [72].

\section{COVID-19}

People are attracted by its much talked off benefits, like: increased appetite, decreased pain and immunosuppresant action to overcome COVID-19. Like tobacco, chronic cannabis smoking can lead to increased sputum production, cough; reduced pulmonary function and respiratory tract diseases. Sharing of cannabis is common social practice in the form of waterpipes (bong, hookah, shisha) which during the pandemic can increase virustransmission risk [74]. Progression of COVID-19 is determined by two phase immune response: first phase related to eliminate the virus and progression to severe form and second phase related to virally induced cytokine storm syndrome. Cannabis compounds containing $\mathrm{CBD}$, cannabigerol (CBG) and tetrahydrocannabivarin (THCV) may have clinical value (anti-inflammatory effect) by reducing cytokine secretion in lung epithelial cells. This is in contrast to fraction from Cannabis sativa Arbel strain $\left(\mathrm{F}_{\mathrm{CBD}}\right)$ which also contains terpenes which is found to increase macrophage 
secreted IL-6 and IL-8 levels. It will ultimately result in worsening of cytokine storm causing more harm in COVID-19 patients [75].

\section{Conclusions}

Despite widespread belief of benefit from medicinal cannabis consumption, we are less aware of its negative consequences. Changing global dynamics moving towards decriminalization and legalization of cannabis and our current understanding of cannabis use in any form (medicinal or recreational) are matter of concern. Analysis of the literature shows more evidence for harms than therapeutic benefits of cannabis in majority of health conditions. This review shows that its benefit lacks adequate evidence warranting for further study. In pretext of already available better options (with better efficacy and safety profile), consideration of this agent cannot be favored. And, its harms and adverse effects are clearly evidence based, mainly from the perspective of mental health effects and serious condition (e.g., cardiac dysrhythmia). Hence, the authors are of the firm opinion that its use should not be suggested and needs serious consideration before using it.

\section{References}

1. Choi S, Huang BC, Gamaldo CE (2020) Therapeutic uses of Cannabis on sleep disorders and related conditions. Journal of Clinical Neurophysiology 37(1): 39-49.

2. (2020) Drug supply.

3. Sulak D (2015) An introduction to the endocannabinoid system. O'Shaughnessy's 16: 3-7.

4. Klumpers LE, Thacker DL (2019) A brief background on cannabis: From plant to medical indications. Journal of AOAC International 102(2): 412420.

5. Kluger B, Triolo P, Jones W, Jankovic J (2015) The therapeutic potential of cannabinoids for movement disorders. Movement disorders 30(3): 313-327.

6. DeZeeuwRA, MalingreTM,MerkusFW(1972) $\Delta 1$-Tetrahydrocannabinolic acid, an important component in the evaluation of cannabis products. Journal of Pharmacy and Pharmacology 24(1): 1-6.

7. (2020) Office of the Commissioner. FDA and Cannabis: Research and Drug Approval Process.

8. Ebbert JO, Scharf EL, Hurt RT (2018) Medical cannabis. In: Mayo Clinic Proceedings 2018 Dec 1. Elsevier 93(12): 1842-1847.

9. (2021) Current Scheduling Recommendations. n.d.

10. Sarris J, Sinclair J, Karamacoska D, Davidson M, Firth J (2020) Medicinal cannabis for psychiatric disorders: a clinically-focused systematic review. BMC psychiatry 20(1): 24.

11. Ashton CH (2001) Pharmacology and effects of cannabis: a brief review. The British Journal of Psychiatry 178(2): 101-6.

12. (2020) Cannabis Use Disorders- Level 4 Cause.

13. (2017) National Academies of Sciences, Engineering, and Medicine. The health effects of cannabis and cannabinoids: the current state of evidence and recommendations for research. National Academies Press.

14. Solimini R, Rotolo MC, Pichini S, Pacifici R (2017) Neurological Disorders in Medical Use of Cannabis: An Update. CNS Neurol Disord Drug Targets 16(5): 527-533.
15. Lattanzi S, Brigo F, Trinka E, Zaccara G, Cagnetti C, et al. (2018) Efficacy and Safety of Cannabidiol in Epilepsy: A Systematic Review and MetaAnalysis. Drugs 78(17): 1791-1804.

16. (2021) Office of the Commissioner. FDA Regulation of Cannabis and Cannabis-Derived Products: Q \& A.

17. Shakya DR (2013) Psychiatric symptoms and disorders in seizure cases referred to psychiatric out-patient service. J Neuroscience and Behavioural Health 5(1): 13-19.

18. Li, Han, Yuzhi Liu, Danni Tian, Lei Tian, Xingke Ju, et al. (2020) Overview of Cannabidiol (CBD) and Its Analogues: Structures, Biological Activities, and Neuroprotective Mechanisms in Epilepsy and Alzheimer's Disease. European Journal of Medicinal Chemistry 192: 112163.

19. GGPeprah K, McCormack S (2019) Medical cannabis for the treatment of dementia: a review of clinical effectiveness and guidelines.

20. Maust DT, Bonar EE, Ilgen MA, Blow FC, Kales HC (2016) Agitation in Alzheimer disease as a qualifying condition for medical marijuana in the United States. The American Journal of Geriatric Psychiatry 24(11): 1000-1003.

21. Chagas MH, Zuardi AW, Tumas V, Pena-Pereira MA, Sobreira ET, et al. (2014) Effects of cannabidiol in the treatment of patients with Parkinson's disease: an exploratory double-blind trial. Journal of Psychopharmacology 28(11): 1088-1098.

22. Moreno JL, Caldentey JG, Cubillo PT, Romero CR, Ribas GG, et al. (2016) A double-blind, randomized, cross-over, placebo-controlled, pilot trial with Sativex in Huntington's disease. Journal of neurology 263(7): 13901400 .

23. Otero-Romero S, Sastre-Garriga J, Comi G, Hartung HP, SoelbergSørensen $P$, et al. (2016) Pharmacological management of spasticity in multiple sclerosis: systematic review and consensus paper. Multiple Sclerosis Journal 22(11): 1386-1396.

24. Zajicek JP, Hobart JC, Slade A, Barnes D, Mattison PG, t al. (2012) Multiple sclerosis and extract of cannabis: results of the MUSEC trial. Journal of Neurology, Neurosurgery \& Psychiatry 83(11): 1125-1132.

25. Collin C, Davies P, Mutiboko IK, Ratcliffe S, Sativex Spasticity in MS Study Group (2007) Randomized controlled trial of cannabis-based medicine in spasticity caused by multiple sclerosis. European journal of neurology 14(3): 290-296

26. Whiting PF, Wolff RF, Deshpande S, Di Nisio M, Duffy S, et al. (2015) Cannabinoids for medical use: a systematic review and meta-analysis. JAMA 313(24): 2456-2473.

27. Singh S, Balhara YP (2017) A review of Indian research on co-occurring cannabis use disorders \& psychiatric disorders. The Indian journal of medical research 146(2): 186.

28. Moore TH, Zammit S, Lingford-Hughes A, Barnes TR, Jones PB, et al. (2007) Cannabis use and risk of psychotic or affective mental health outcomes: a systematic review. The Lancet 370(9584): 319-328.

29. Van Winkel R, Kuepper R (2014) Epidemiological, neurobiological, and genetic clues to the mechanisms linking cannabis use to risk for nonaffective psychosis. Annual review of clinical psychology 10(1): 767791.

30. Schoeler T, Monk A, Sami MB, Klamerus E, Foglia E, et al. (2016) Continued versus discontinued cannabis use in patients with psychosis: a systematic review and meta-analysis. The Lancet Psychiatry 3(3): 215225 .

31. Starzer MS, Nordentoft M, Hjorthøj C (2018) Rates and predictors of conversion to schizophrenia or bipolar disorder following substanceinduced psychosis. American Journal of Psychiatry 175(4): 343-350.

32. Lowe DJ, Sasiadek JD, Coles AS, George TP (2019) Cannabis and mental illness: a review. European archives of psychiatry and clinical neuroscience 269(1): 107-120. 
33. Onaemo VN, Fawehinmi TO, D’Arcy C (2021) Comorbid Cannabis Use Disorder with Major Depression and Generalized Anxiety Disorder: A Systematic Review and Meta-Analyses of nationally representative epidemiological surveys. Journal of affective disorders 281: 467-475.

34. Esmaeelzadeh S, Moraros J, Thorpe L, Bird Y (2018) Examining the association and directionality between mental health disorders and substance use among adolescents and young adults in the US and Canada- a systematic review and meta-analysis. Journal of clinical medicine 7(12): 543.

\section{5. (2021) EPIDIOLEX ${ }^{\circledR}$ (cannabidiol).}

36. Gibbs M, Winsper C, Marwaha S, Gilbert E, Broome M, et al. (2015) Cannabis use and mania symptoms: a systematic review and metaanalysis. Journal of affective disorders 171: 39-47.

37. Feingold D, Weiser M, Rehm J, Lev-Ran S (2015) The association between cannabis use and mood disorders: A longitudinal study. Journal of affective disorders 172: 211-218.

38. Bally N, Zullino D, Aubry JM (2014) Cannabis use and first manic episode. Journal of affective disorders 165: 103-108.

39. Kedzior KK, Laeber LT (2014) A positive association between anxiety disorders and cannabis use or cannabis use disorders in the general population-a meta-analysis of 31 studies. BMC psychiatry 14(1): 1-22.

40. Xue S, Husain MI, Zhao H, Ravindran AV (2020) Cannabis Use and Prospective Long-Term Association with Anxiety: A Systematic Review and Meta-Analysis of Longitudinal Studies: Usage du cannabis et association prospective à long terme avec l'anxiété: une revue systématique et uneméta-analysed'étudeslongitudinales. The Canadian Journal of Psychiatry 66(2).

41. Upadhyaya KD (2004) Cannabis Abuse and Psychiatric Morbidity. Nepal Med Assoc 43: 91-93.

42. Shakya DR (2020) Comorbidities in Nepalese psychiatry out-patients with Obsessive Compulsive Disorder. J Psychiatrists' Assoc. Nepal 9(1): 34-40.

43. Colizzi M, Bhattacharyya S (2017) Does cannabis composition matter? Differential effects of delta-9-tetrahydrocannabinol and cannabidiol on human cognition. Current Addiction Reports 4(2): 62-74.

44. Scott JC, Slomiak ST, Jones JD, Rosen AF, Moore TM, et al. (2018) Association of cannabis with cognitive functioning in adolescents and young adults: a systematic review and meta-analysis. JAMA psychiatry 75(6): 585-595.

45. Meier MH, Caspi A, Ambler A, Harrington H, Houts R, et al. (2012) Persistent cannabis users show neuropsychological decline from childhood to midlife. Proceedings of the National Academy of Sciences 109(40): 2657-2664

46. Sharma B (1975) Cannabis and its Users in Nepal. British Journal of Psychiatry 127(6): 550-552.

47. Meier MH (2021) Cannabis use and psychosocial functioning: evidence from prospective longitudinal studies. Current Opinion in Psychology 38: $19-24$.

48. Aviram J, Samuelly-Leichtag G (2017) Efficacy of Cannabis-Based Medicines for Pain Management: A Systematic Review and Meta-Analysis of Randomized Controlled Trials. Pain Physician 20(6): 755-796.

49. Orrego-González E, Londoño-Tobón L, Ardila-González J, Polania-Tovar D, Valencia-Cárdenas A, et al. (2020) Cannabinoid Effects on Experimental Colorectal Cancer Models Reduce Aberrant Crypt Foci (ACF) and Tumor Volume: A Systematic Review. Evidence-Based Complementary and Alternative Medicine 2020(5): 1-13.

50. Gurney J, Shaw C, Stanley J, Signal V, Sarfati D (2015) Cannabis exposure and risk of testicular cancer: a systematic review and meta-analysis. BMC cancer 15(1): 1-10.
51. Richards JR (2020) Mechanisms for the Risk of Acute Coronary Syndrome and Arrhythmia Associated With Phytogenic and Synthetic Cannabinoid Use. Journal of Cardiovascular Pharmacology and Therapeutics 25(6): 508-522.

52. Richards JR, Blohm E, Toles KA, Jarman AF, Ely DF, et al. (2020) The association of cannabis use and cardiac dysrhythmias: a systematic review. Clinical Toxicology 58(1): 861-869.

53. Patel RS, Kamil SH, Bachu R, Adikey A, Ravat V, et al. (2020) Marijuana use and acute myocardial infarction: a systematic review of published cases in the literature. Trends in cardiovascular medicine 30(5): 298307.

54. Karasu T, Marczylo TH, Maccarrone M, Konje JC (2011) The role of sex steroid hormones, cytokines and the endocannabinoid system in female fertility. Human reproduction update 17(3): 347-361.

55. Gundersen TD, Jørgensen N, Andersson AM, Bang AK, Nordkap L, et al. (2015) Association between use of marijuana and male reproductive hormones and semen quality: a study among 1,215 healthy young men. American journal of epidemiology 182(6): 473-481.

56. Nassan FL, Arvizu M, Mínguez-Alarcón L, Williams PL, Attaman J, et al. (2019) Marijuana smoking and markers of testicular function among men from a fertility centre. Human Reproduction 34(4): 715-723.

57. Jukic AM, Weinberg CR, Baird DD, Wilcox AJ (2007) Lifestyle and reproductive factors associated with follicular phase length. Journal of women's health 16(9): 1340-1347.

58. Gunn JK, Rosales CB, Center KE, Nuñez A, Gibson SJ, et al. (2016) Prenatal exposure to cannabis and maternal and child health outcomes: a systematic review and meta-analysis. BMJ open 6(4).

59. Karila L, Cazas O, Danel T, Reynaud M (2006) Short-and long-term consequences of prenatal exposure to cannabis. Journal de gynecologie, obstetrique et biologie de la reproduction 35(1): 62-70.

60. Schwitzer T, Schwan R, Angioi-Duprez K, Giersch A, Laprevote V (2016) The endocannabinoid system in the retina: from physiology to practical and therapeutic applications. Neural plasticity 2016(5): 1-10.

61. Kokona D, Georgiou PC, Kounenidakis M, Kiagiadaki F, Thermos K (2016) Endogenous and Synthetic Cannabinoids as Therapeutics in Retinal Disease. Neural Plast 2016(8-9): 1-12.

62. Passani A, Posarelli C, Sframeli AT, Perciballi L, Pellegrini M, et al. (2020) Cannabinoids in Glaucoma Patients: The Never-Ending Story. Journal of clinical medicine 9(12): 3978.

63. Cairns EA, Baldridge WH, Kelly ME (2016) The endocannabinoid system as a therapeutic target in glaucoma. Neural plasticity 2016(2): 1-10.

64. Thapa D, Cairns EA, Szczesniak AM, Toguri JT, Caldwell MD, et al. (2018) The cannabinoids $\triangle 8 \mathrm{THC}, \mathrm{CBD}$, and HU-308 act via distinct receptors to reduce corneal pain and inflammation. Cannabis and cannabinoid research 3(1): 11-20.

65. Polat N, Cumurcu B, Cumurcu T, Tuncer I (2018) Corneal endothelial changes in long-term cannabinoid users. Cutan Ocul Toxicol 37(1): 1923.

66. Perisetti A, Gajendran M, Dasari CS, Bansal P, Aziz M, et al. (2020) Cannabis hyperemesis syndrome: an update on the pathophysiology and management. Annals of Gastroenterology 33(6): 571.

67. A Galli J, Andari Sawaya R, K Friedenberg F (2011) Cannabinoid hyperemesis syndrome. Current drug abuse reviews 4(4): 241-249.

68. Peprah K, McCormack S (2019) Medical Cannabis for the Treatment of Dementia: A Review of Clinical Effectiveness and Guidelines. Ottawa (ON): Canadian Agency for Drugs and Technologies in Health.

69. Ramaekers JG, Berghaus G, van Laar M, Drummer OH (2004) Dose related risk of motor vehicle crashes after cannabis use. Drug and alcohol dependence 73(2): 109-119. 
70. Bondallaz P, Favrat B, Chtioui H, Fornari E, Maeder P, et al. (2016) Cannabis and its effects on driving skills. Forensic science international 268: 92-102.

71. Goullé JP, Guerbet M (2014) Tetrahydrocannabinol pharmacokinetics; new synthetic cannabinoids; road safety and cannabis. Bulletin de L'academie Nationale de Medecine 198(3): 541-546.

72. Babson KA, Sottile J, Morabito D (2017) Cannabis, cannabinoids, and sleep: a review of the literature. Current psychiatry reports 19(4): 1-2.

73. Winsler A, Deutsch A, Vorona RD, Payne PA, Szklo-Coxe M (2015) Sleepless in Fairfax: the difference one more hour of sleep can make for

ISSN: 2574-1241

DOI: $10.26717 /$ BJSTR.2021.36.005886

Dhana Ratna Shakya. Biomed J Sci \& Tech Res

(c) (P) This work is licensed under Creative

Submission Link: https://biomedres.us/submit-manuscript.php teen hopelessness, suicidal ideation, and substance use. Journal of youth and adolescence 44(2): 362-378.

74. Pastor FP, Folgar MI, Carvalho N, Carvalho F, Horcajadas FA (2020) Therapeutic Cannabis and COVID-19: between opportunism and infoxication Cannabis terapéutico y COVID-19: entre el oportunismo y la infoxicación. adicciones 32(3): 167-172.

75. Anil SM, Shalev N, Vinayaka AC, Nadarajan S, Namdar D, et al. (2021) Cannabis compounds exhibit anti-inflammatory activity in vitro in COVID-19-related inflammation in lung epithelial cells and proinflammatory activity in macrophages. Scientific reports 11(1): 1-4.

$\begin{array}{ll}\text { BIOMEDICAL } & \text { Assets of Publishing with us } \\ \text { RESEARCHES } & \text { - Global archiving of articles } \\ \text { - Immediate, unrestricted online access } & \text { - Rigorous Peer Review Process } \\ & \text { - Authors Retain Copyrights } \\ & \end{array}$

